$\mathrm{e}^{+} \mathrm{e}^{-}$Collisions from Phi to Psi 2013 (PHIPSI2013)

International Journal of Modern Physics: Conference Series

Vol. 35 (2014) 1460440 (8 pages)

(C) The Author

DOI: $10.1142 /$ S2010194514604402

\title{
RECENT $\tau$ LEPTON RESULTS FROM BABAR
}

\author{
ALBERTO LUSIANI \\ Classe di Scienze, Scuola Normale Superiore, piazza dei Cavalieri 7 \\ 56125 Pisa, Italy \\ alberto.lusiani@pi.infn.it
}

Published 18 December 2014

\begin{abstract}
We report recent measurements on $\tau$ leptons obtained by the BABAR collaboration using the entire recorded sample of electron-positron collisions at and around the $\Upsilon(4 S$ ) (about $\left.470 \mathrm{fb}^{-1}\right)$. The events were recorded at the PEP-II asymmetric collider at the SLAC National Accelerator Laboratory. The measurements include high multiplicity $\tau$ decay branching fractions with 3 or 5 charged particles in the final state, a search for the second class current the $\tau$ decay $\tau \rightarrow \pi \eta^{\prime} \nu, \tau$ branching fractions into final states containing two $K_{S}$ mesons, $\tau^{-} \rightarrow h^{-} K_{S}^{0} K_{S}^{0}\left(\pi^{0}\right) \nu_{\tau}$, with $h=\pi, K$, and preliminary measurements of hadronic spectra of $\tau$ decays with three hadrons $\left(\tau^{-} \rightarrow h^{-} h^{+} h^{-} \nu_{\tau}\right.$ decays, where $h=\pi, K)$. The results improve the experimental knowledge of the $\tau$ lepton properties and can be used to improve the precision tests of the Standard Model.
\end{abstract}

Keywords: Tau babar.

PACS Numbers: 13.35.Dx, 14.60.Fg

\section{Introduction}

LEP experiments, particularly ALEPH, have published measurements on the largest $\tau$ branching fractions up to a level of precision that B-factories were not yet able to match. B-factories however have been able to improve the experimental precision of measurements and searches for smaller $\tau$ branching fractions.

We report in the following on recent measurements by the BABAR collaboration, relying on data recorded at the PEP-II asymmetric-energy $e^{+} e^{-}$storage rings operated at the SLAC National Accelerator Laboratory. About 430 million $\tau$ pairs have been studied, corresponding to an integrated luminosity $\mathcal{L}=424 \mathrm{fb}^{-1}$ at a center-of-mass (CM) energy of $10.58 \mathrm{GeV}$ and $44 \mathrm{fb}^{-1}$ at $10.54 \mathrm{GeV}$.

This is an Open Access article published by World Scientific Publishing Company. It is distributed under the terms of the Creative Commons Attribution 3.0 (CC-BY) License. Further distribution of this work is permitted, provided the original work is properly cited. 

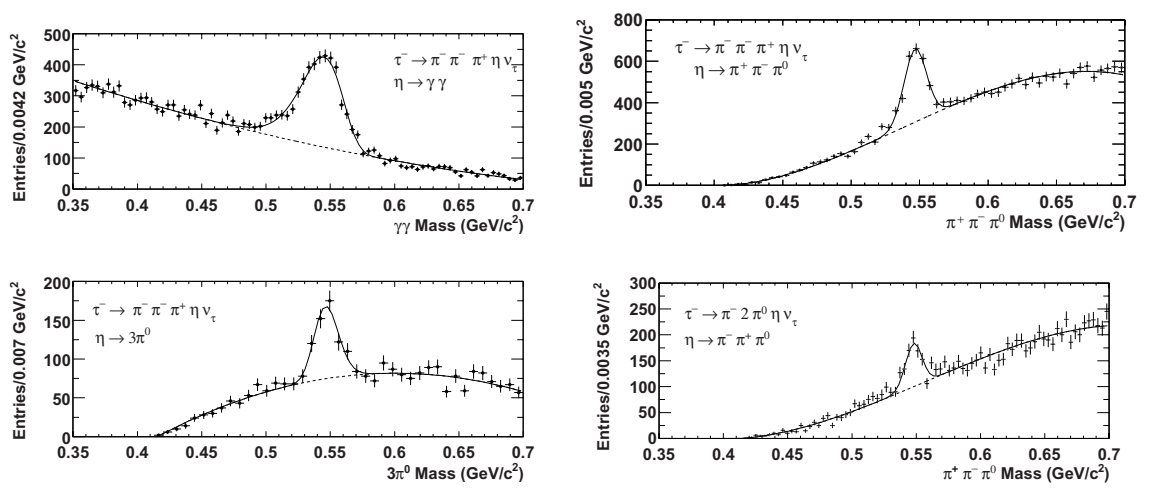

Fig. 1. The $\gamma \gamma, \pi^{+} \pi^{-} \pi^{0}$, and $3 \pi^{0}$ invariant mass distributions for $\tau^{-} \rightarrow \pi^{-} \pi^{+} \pi^{-} \eta \nu_{\tau}$ candidates, and the $\pi^{+} \pi^{-} \pi^{0}$ invariant mass distribution for $\tau^{-} \rightarrow \pi^{-} 2 \pi^{0} \eta \nu_{\tau}$ decay candidates, after all selection criteria are applied. The solid lines represent the fit to the $\eta$ peak and background. The dashed lines show the extrapolation of the background function under the $\eta$ peak.

\section{High multiplicity $\tau$ decays}

The BABAR Collaboration has published a collection of results on high multiplicity $\tau$ decays. ${ }^{1}$ Reconstructed events tracks are split in two hemispheres defined by the thrust direction, and candidate events are selected requiring one track in one hemisphere (tag-side) and 3 or 5 tracks in the other one (signal side). To reduce backgrounds from dileptons, the tag-side track momentum must be smaller than $4 \mathrm{GeV} / c$. To suppress low multiplicity $q \bar{q}$ events, the tag-side track must be an identified electron or muon and the tag-side hemisphere must contain either zero or at most one energetic $(E>1 \mathrm{GeV})$ electromagnetic calorimeter cluster, Neutral pion candidates are reconstructed in the signal hemisphere from photon pairs with the appropriate invariant mass.

The selection efficiencies are determined with signal Monte Carlo samples, which are processed in the same manner as data. Monte Carlo simulated events are also used to estimate and subtract backgrounds. The Monte Carlo is calibrated to match the data for low-multiplicity $q \bar{q}$ backgrounds by studying control samples with a modified selection where the signal side invariant mass is larger than the $\tau$ mass.

\subsection{High multiplicity $\tau$ decays into hadronic resonances}

\subsection{1. $\tau^{-} \rightarrow 3(\pi)^{-} \eta \nu_{\tau}$}

The selected high multiplicity $\tau$ decay candidates contain the following final states with the $\eta$ meson: $\pi^{-} \pi^{+} \pi^{-} \eta \nu_{\tau}\left(\eta \rightarrow \gamma \gamma, \eta \rightarrow \pi^{-} \pi^{+} \pi^{0}\right)$ and $\rightarrow 3 \pi^{0} \eta \nu_{\tau}(\eta \rightarrow$ $\left.\pi^{-} \pi^{+} \pi^{0}\right)$. The signal yield is determined by fitting the $\eta$ mass peak in the invariant mass distributions (Fig. 1). All three determinations of the $\tau^{-} \rightarrow \pi^{-} \pi^{+} \pi^{-} \eta \nu_{\tau}$ branching fraction are statistically consistent, and an average is computed:

$$
B\left(\tau^{-} \rightarrow \pi^{-} \pi^{+} \pi^{-} \eta \nu_{\tau}\right)=(2.25 \pm 0.07 \pm 0.12) \times 10^{-4} .
$$


The $\tau^{-} \rightarrow \pi^{-} 2 \pi^{0} \eta \nu_{\tau}$ branching fraction on the other hand is found to be:

$$
B\left(\tau^{-} \rightarrow \pi^{-} 2 \pi^{0} \eta \nu_{\tau}\right)=(2.01 \pm 0.34 \pm 0.22) \times 10^{-4} .
$$

The ratio of the $\tau^{-} \rightarrow \pi^{-} \pi^{+} \pi^{-} \eta \nu_{\tau}$ to $\tau^{-} \rightarrow \pi^{-} 2 \pi^{0} \eta \nu_{\tau}$ branching fractions is expected to be two if the decay is dominated by the $\tau^{-} \rightarrow \pi^{-} f_{1} \nu_{\tau}$ decay mode, but the data do not support this hypothesis. The measurements are in good agreement with the results from the CLEO Collaboration, $(2.3 \pm 0.5) \times 10^{-4}$ and $(1.5 \pm 0.5) \times$ $10^{-4}$, for $\tau^{-} \rightarrow \pi^{-} \pi^{+} \pi^{-} \eta \nu_{\tau}$ and $\tau^{-} \rightarrow \pi^{-} 2 \pi^{0} \eta \nu_{\tau}$, respectively. ${ }^{2}$

\subsection{2. $\tau^{-} \rightarrow \pi^{-} f_{1} \nu_{\tau}$}

The branching fraction of $\tau^{-} \rightarrow \pi^{-} f_{1} \nu_{\tau}$ as well as the $f_{1}$ mass are measured using the $f_{1} \rightarrow 2 \pi^{+} 2 \pi^{-}$and $f_{1} \rightarrow \pi^{+} \pi^{-} \eta$ decay modes, where $\eta$ is reconstructed using $\eta \rightarrow \gamma \gamma, \eta \rightarrow \pi^{+} \pi^{-} \pi^{0}$, and $\eta \rightarrow 3 \pi^{0}$ events. The numbers of $\tau^{-} \rightarrow \pi^{-} f_{1} \nu_{\tau}$ candidates are determined by fitting the $f_{1}$ peak in the $2 \pi^{+} 2 \pi^{-}$and $\pi^{+} \pi^{-} \eta$ invariant mass distributions. The product of the $\tau^{-} \rightarrow \pi^{-} f_{1} \nu_{\tau}$ and $f_{1} \rightarrow 2 \pi^{+} 2 \pi^{-}$branching fractions, and the product of the $\tau^{-} \rightarrow \pi^{-} f_{1} \nu_{\tau}$ and $f_{1} \rightarrow \pi^{+} \pi^{-} \eta$ branching fractions, are measured to be

$$
\begin{gathered}
B\left(\tau^{-} \rightarrow \pi^{-} f_{1} \nu_{\tau}\right) B\left(f_{1} \rightarrow 2 \pi^{+} 2 \pi^{-}\right)=(5.20 \pm 0.31 \pm 0.37) \times 10^{-5}, \\
B\left(\tau^{-} \rightarrow \pi^{-} f_{1} \nu_{\tau}\right) B\left(f_{1} \rightarrow \pi^{+} \pi^{-} \eta\right)=(1.26 \pm 0.06 \pm 0.06) \times 10^{-4},
\end{gathered}
$$

respectively, where the second result is the weighted average of the three $\eta$ modes. The $B\left(\tau^{-} \rightarrow \pi^{-} f_{1} \nu_{\tau}\right)$ branching fraction is determined to be $(4.73 \pm 0.28 \pm 0.45) \times$ $10^{-4}$ and $(3.60 \pm 0.18 \pm 0.23) \times 10^{-4}$, as obtained by dividing the product branching fractions by $B\left(f_{1} \rightarrow 2 \pi^{+} 2 \pi^{-}\right)=0.110_{-0.006}^{+0.007}$ and $B\left(f_{1} \rightarrow \pi^{+} \pi^{-} \eta\right)=0.349_{-0.015}^{+0.013}{ }^{3}$ respectively. The two values branching fraction are consistent with each other to within two standard deviations of the combined statistical and systematic uncertainties. The ratio of the product branching fractions is used to determine the ratio of the $f_{1} \rightarrow 2 \pi^{+} 2 \pi^{-}$and $f_{1} \rightarrow \pi^{+} \pi^{-} \eta$ branching fractions as

$$
\frac{\mathcal{B}\left(f_{1} \rightarrow 2 \pi^{+} 2 \pi^{-}\right)}{B\left(f_{1} \rightarrow \pi \pi \eta\right)}=0.28 \pm 0.02 \pm 0.02,
$$

where $\mathcal{B}\left(f_{1} \rightarrow \pi \pi \eta\right)=1.5 \times \mathcal{B}\left(f_{1} \rightarrow \pi^{+} \pi^{-} \eta\right)$ based on isospin symmetry. This agrees with the average value of $0.41 \pm 0.14$ quoted by the Particle Data Group ${ }^{3}$ but disagrees with their fit value of $0.63 \pm 0.06 .^{3}$

Just for the $f_{1}$ mass measurement, the sample is enlarged by releasing the requirements on photons and lepton identification in the tag hemisphere. The $f_{1}$ mass is determined by fitting the peak with a non-relativistic Breit-Wigner function, which was used in previous measurements of the $f_{1}$ mass. ${ }^{3}$ For each decay mode, simulated events are use to determine the measurement bias to be subtracted, due to the selection and fitting procedures. We determine the mass of the $f_{1}(1258)$ meson to be

$$
M_{f_{1}}=(1.28116 \pm 0.00039 \pm 0.00045) \mathrm{GeV} / \mathrm{c}^{2} .
$$




\section{A. Lusiani}

The systematic uncertainty includes the calibration bias uncertainty. This result is in good agreement with the value $(1.2818 \pm 0.0006) \mathrm{GeV} / \mathrm{c}^{2}{ }^{3}{ }^{3}$

\subsection{3. $\tau^{-} \rightarrow 3(\pi)^{-} \omega \nu_{\tau}$}

We measure the $\tau^{-} \rightarrow \pi^{+} 2 \pi^{-} \omega \nu_{\tau}$ and $\tau^{-} \rightarrow \pi^{-} 2 \pi^{0} \omega \nu_{\tau}$ branching fractions by fitting the $\omega$ peak in the $\pi^{+} \pi^{-} \pi^{0}$ invariant mass distributions with a Breit-Wigner distribution, which is convolved with a Gaussian distribution to take into account the detector resolution. The $\tau^{-} \rightarrow \pi^{-} 2 \pi^{0} \omega \nu_{\tau}$ sample has substantial contributions from $\tau^{-} \rightarrow \pi^{-} \omega \nu_{\tau}$ and $\tau^{-} \rightarrow \pi^{-} \pi^{0} \omega \nu_{\tau}$ decays. The background is estimated with the Monte Carlo simulation and verified using data and simulation control samples. The control samples follow the nominal selection criteria but select one or two $\pi^{0}$ instead of three $\pi^{0}$ mesons. The branching fractions are found to be

$$
\begin{aligned}
B\left(\tau^{-} \rightarrow \pi^{+} 2 \pi^{-} \omega \nu_{\tau}\right) & =(8.4 \pm 0.4 \pm 0.6) \times 10^{-5}, \\
B\left(\tau^{-} \rightarrow \pi^{-} 2 \pi^{0} \omega \nu_{\tau}\right) & =(7.3 \pm 1.2 \pm 1.2) \times 10^{-5} .
\end{aligned}
$$

\subsection{Non-resonant high multiplicity $\tau$ decays}

A fraction of the $\tau$ decay width into the selected final states proceeds outside the considered resonances. We do not attempt to identify additional resonances and we measure inclusive "non-resonant" branching fractions by subtracting the identified resonant contributions from the total branching fractions.

The decay $\tau^{-} \rightarrow \pi^{+} 2 \pi^{-} 3 \pi^{0} \nu_{\tau}$ appears to proceed exclusively via resonances, and its non-resonant branching fraction is measured to be consistent with zero $\left[(1.0 \pm 0.8 \pm 3.0) \times 10^{-5}\right]$. The corresponding all-inclusive branching fraction on the other hand is $B\left(\tau^{-} \rightarrow \pi^{+} 2 \pi^{-} 3 \pi^{0} \nu_{\tau}\right)=(2.07 \pm 0.18 \pm 0.37) \times 10^{-4}$.

The decay $\tau^{-} \rightarrow 2 \pi^{+} 3 \pi^{-} \nu_{\tau}$ proceeds predominantly in a non-resonant manner and its non-resonant branching fraction is determined to be $B\left(\tau^{-} \rightarrow 2 \pi^{+} 3 \pi^{-} \nu_{\tau}\right)=$ $(7.68 \pm 0.04 \pm 0.40) \times 10^{-4}$. The resonant component $\tau^{-} \rightarrow \pi^{+} 2 \pi^{-} \omega \nu_{\tau}\left(\omega \rightarrow \pi^{+} \pi^{-} \gamma\right)$ is subtracted to obtain the above measurement. The inclusive branching fraction is $\left(\tau^{-} \rightarrow 2 \pi^{+} 3 \pi^{-} \nu_{\tau}\right)=(8.33 \pm 0.04 \pm 0.43) \times 10^{-4}$ and includes the resonant contribution $\tau^{-} \rightarrow \pi^{-} f_{1} \nu_{\tau}$ with $f_{1} \rightarrow 2 \pi^{+} 2 \pi^{-}$.

The $\tau^{-} \rightarrow 2 \pi^{+} 3 \pi^{-} \pi^{0} \nu_{\tau}$ decays are dominated by the resonant modes. We determine the non-resonant fraction to be $B\left(\tau^{-} \rightarrow 2 \pi^{+} 3 \pi^{-} \pi^{0} \nu_{\tau}\right)=(3.6 \pm$ $0.3 \pm 0.9) \times 10^{-5}$. The inclusive branching fraction is $B\left(\tau^{-} \rightarrow 2 \pi^{+} 3 \pi^{-} \pi^{0} \nu_{\tau}=\right.$ $(1.65 \pm 0.05 \pm 0.09) \times 10^{-4}$ and is obtained by adding the non-resonant branching fraction and the resonant branching fractions attributed to the $\tau \rightarrow 2 \pi^{-} \pi^{+} \eta \nu_{\tau}$ via $\eta \rightarrow \pi^{+} \pi^{-} \pi^{0}$ and $\tau \rightarrow 2 \pi^{-} \pi^{+} \omega \nu_{\tau}$ via $\omega \rightarrow \pi^{+} \pi^{-} \pi^{0}$ decays.

\subsection{5-prong $\tau$ decays with kaons}

No evidence is found for high-multiplicity $\tau$ decays with one charged kaon or or two oppositely charged kaons and 90\% CL upper limits are set in the range 
0.45-2.4 $\times 10^{-6}$. There are no theoretical predictions for these modes. We estimate that $B\left(\tau^{-} \rightarrow K^{-} 2 \pi^{-} 2 \pi^{+} \nu_{\tau}\right) \sim 10^{-5}-10^{-6}$ if the decay is related to $B\left(\tau^{-} \rightarrow 3 \pi^{-} 2 \pi^{+} \nu_{\tau}\right)$ by the ratio of the CKM matrix elements $\left(V_{u s} / V_{u d}\right)$.

\section{Search for $2^{\text {nd }}$ class current decays}

We search for the second-class current decay $\tau^{-} \rightarrow \pi^{-} \eta^{\prime}(958) \nu_{\tau}$ (with $\eta^{\prime} \rightarrow \pi^{+} \pi^{-} \eta$ ) and in the process we also measure the branching fraction of the related firstclass channels $\tau^{-} \rightarrow \pi^{-} \pi^{0} \eta^{\prime}(958) \nu_{\tau}$ and $\tau^{-} \rightarrow K^{-} \eta^{\prime}(958) \nu_{\tau}$, which are potential background sources. We find no evidence for any of these decays and place the following upper limits on the branching fractions at the $90 \%$ confidence level:

$$
\begin{gathered}
B\left(\tau^{-} \rightarrow \pi^{-} \pi^{0} \eta^{\prime}(958) \nu_{\tau}\right)<1.2 \times 10^{-5}, \\
B\left(\tau^{-} \rightarrow K^{-} \eta^{\prime}(958) \nu_{\tau}\right)<2.4 \times 10^{-6}, \\
B\left(\tau^{-} \rightarrow \pi^{-} \eta^{\prime}(958) \nu_{\tau}\right)<4.0 \times 10^{-6} .
\end{gathered}
$$

The second-class current branching fraction is expected ${ }^{4}$ to be smaller than $1.4 \times$ $10^{-6}$.

\section{The branching fraction of $\tau^{-} \rightarrow h^{-} K_{S}^{0} K_{S}^{0}\left(\pi^{0}\right) \nu_{\tau}$ decays}

With an analysis similar to the one for high multiplicity decays, we study events with two $K_{S}^{0}$ mesons in the final state, ${ }^{5}$ in two channels that differ by the presence of an additional $\pi^{0} . K_{S}^{0}$ candidates are defined as a pair of oppositely charged pion candidate tracks, with an invariant mass between 0.475 and $0.525 \mathrm{GeV} / \mathrm{c}^{2}$. The branching fractions of the two charged pion modes are determined simultaneously to take into account the cross feed of each decay mode into the other sample.

The $\tau^{-} \rightarrow \pi^{-} K_{S}^{0} K_{S}^{0} \nu_{\tau}$ decay is simulated with Tauola ${ }^{6}$ using $\tau^{-} \rightarrow K^{*-} K^{0} \nu_{\tau}$. The $\tau^{-} \rightarrow \pi^{-} K_{S}^{0} K_{S}^{0} \pi^{0} \nu_{\tau}$ decay is simulated with EvtGen ${ }^{7}$ using $\tau^{-} \rightarrow K^{*-} K^{0} \pi^{0} \nu_{\tau}$ and $\tau^{-} \rightarrow \pi^{-} K^{* 0} K^{0} \nu_{\tau}$. As the simulation does not precisely reproduce the data hadronic invariant mass distributions for the $\tau^{-} \rightarrow h^{-} K_{S}^{0} K_{S}^{0} \nu_{\tau}$ channel, we have weighted the Tauola events to match the data distribution for the $K_{S}^{0} \pi^{-}$invariant mass of Fig. 2. Plots in the following use the re-weighted simulation.

We measure the branching fractions to be:

$$
\begin{aligned}
B\left(\tau^{-} \rightarrow \pi^{-} K_{S}^{0} K_{S}^{0} \nu_{\tau}\right) & =(2.31 \pm 0.04 \pm 0.08) \times 10^{-4} \\
B\left(\tau^{-} \rightarrow \pi^{-} K_{S}^{0} K_{S}^{0} \pi^{0} \nu_{\tau}\right) & =(1.60 \pm 0.20 \pm 0.22) \times 10^{-5}
\end{aligned}
$$

respectively. Fig. 3 reports some measured and simulated invariant hadronic mass distributions for the both channels.

\section{5. $\tau^{-} \rightarrow h^{-} h^{+} h^{-} \nu_{\tau}$ invariant mass spectra}

The BABAR collaboration has presented preliminary results on the measurement of hadronic spectra for $\tau$ decays into three hadrons and a neutrino. For the event 

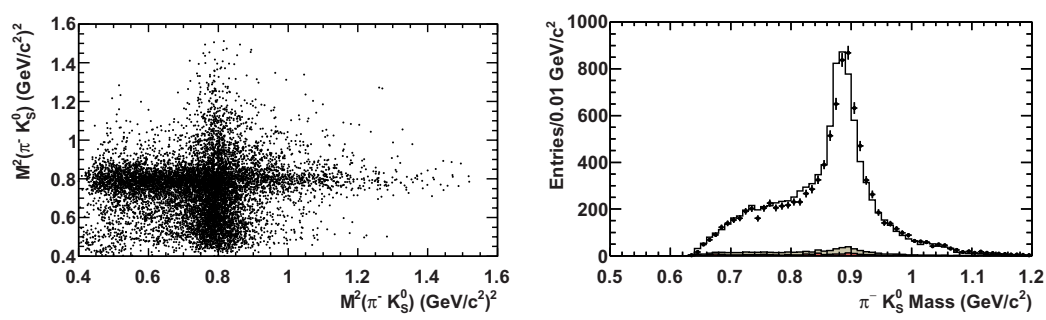

Fig. 2. Dalitz plot and invariant mass distributions of the $K_{S}^{0} \pi^{-}$system for selected $\tau^{-} \rightarrow$ $\pi^{-} K_{S}^{0} K_{S}^{0} \nu_{\tau}$ candidates. events. Both plots contain two entries per event. The points are data and the histograms are the prediction of the Monte Carlo simulation. The plot reports a barely visible simulated background contamination.
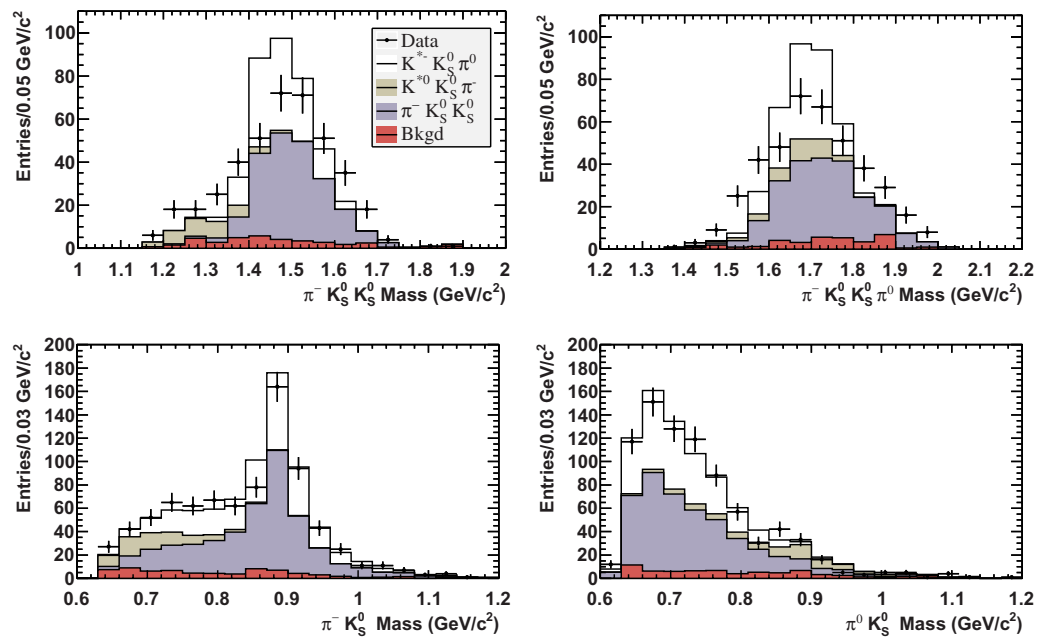

Fig. 3. The $\left(\pi^{-} K_{S}^{0} K_{S}^{0}\right),\left(\pi^{-} K_{S}^{0} K_{S}^{0} \pi^{0}\right),\left(\pi^{-} K_{S}^{0}\right)$, and $\left(\pi^{0} K_{S}^{0}\right)$ invariant mass distributions that pass the selection criteria. The points are data and the histograms are the predictions of the Monte Carlo simulation. The two signal channels are shown in the white $\left(\tau^{-} \rightarrow K^{*-} K^{0} \pi^{0} \nu_{\tau}\right)$ and beige $\left(\tau^{-} \rightarrow K^{* 0} K^{0} \pi^{-} \nu_{\tau}\right)$ histograms. The dark blue histogram is $\tau^{-} \rightarrow \pi^{-} K_{S}^{0} K_{S}^{0} \nu_{\tau}\left(\tau^{-} \rightarrow K^{*-} K^{0} \nu_{\tau}\right)$ decays. The red histogram is the $q \bar{q}$ background.

selection, a sample of $\tau^{-} \rightarrow h^{-} h^{+} h^{-} \nu_{\tau}$ decays events is selected by requiring the partner $\tau^{+}$to decay leptonically. Within this sample, each of the mesons is uniquely identified as a charged pion or kaon, and the decay categorized as $\tau^{-} \rightarrow \pi^{-} \pi^{+} \pi^{-} \nu_{\tau}$, $\tau^{-} \rightarrow K^{-} \pi^{+} \pi^{-} \nu_{\tau}, \tau^{-} \rightarrow K^{-} K^{+} \pi^{-} \nu_{\tau}$ or $\tau^{-} \rightarrow K^{-} K^{+} K^{-} \nu_{\tau}$, where events with $K_{S}^{0}$ have been excluded. After events are selected, the invariant mass distributions are analyzed. The $\tau^{-} \rightarrow h^{-} h^{+} h^{-} \nu_{\tau}$ cross-feed backgrounds between the channels caused by particle mis-identification are measured simultaneously in this analysis. The cross-feed backgrounds are estimated to be $(0.85 \pm 0.01) \%$ for the $\pi^{-} \pi^{+} \pi^{-}$ channel, $(38.5 \pm 0.2) \%$ for the $K^{-} \pi^{+} \pi^{-}$channel, $(2.9 \pm 0.1) \%$ for the $K^{-} K^{+} \pi^{-}$ channel and $(27.7 \pm 3.0) \%$ for the $K^{-} K^{+} K^{-}$channel, where the uncertainties are 

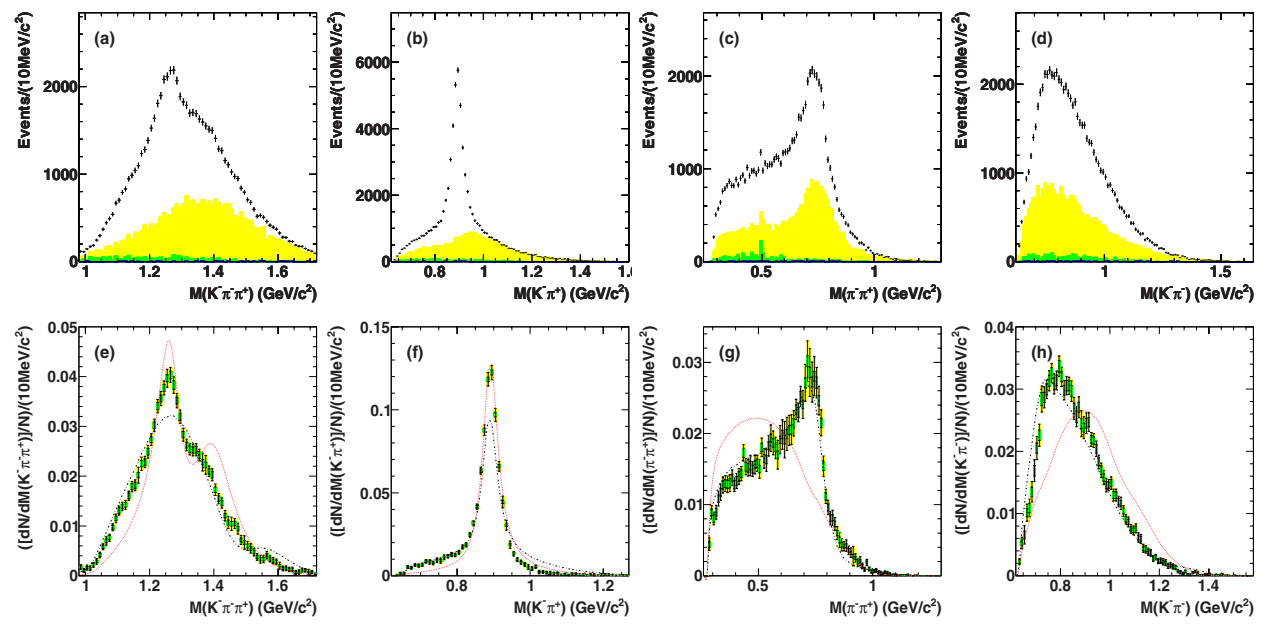

Fig. 4. The reconstructed and unfolded invariant mass spectra for the $\tau^{-} \rightarrow K^{-} \pi^{+} \pi^{-} \nu_{\tau}$ channel. For the reconstructed mass plots, the data is represented by the points with the error bars representing the statistical uncertainty. The blue (dark) histogram represents the non- $\tau$ background MC, the green (medium dark) histogram represents the $\tau$ backgrounds excluding the $\tau^{-} \rightarrow K^{-} \pi^{+} \pi^{-} \nu_{\tau}$ cross-feed which are represented by the yellow (light) histogram. For the unfolded mass plots, the data is represented by the points with the inner error bars (green) representing the statistical uncertainty and the outer error bars (yellow) representing the statistical and systematic uncertainties. The black dashed line is the generator level Monte Carlo distribution used in the BaBar simulation. The red dotted line is the CLEO tune for Tauola $2.8 .^{6}$

from MC statistics. The background fractions from events with an extra $\pi^{0}$ in the candidate samples are estimated to be $(3.6 \pm 0.3) \%$ from $\tau^{-} \rightarrow \pi^{-} \pi^{+} \pi^{-} \pi^{0} \nu_{\tau}$ in $\tau^{-} \rightarrow \pi^{-} \pi^{+} \pi^{-} \nu_{\tau},(2.3 \pm 0.4) \%$ from $\tau^{-} \rightarrow K^{-} \pi^{+} \pi^{-} \pi^{0} \nu_{\tau}$ in $\tau^{-} \rightarrow K^{-} \pi^{+} \pi^{-} \nu_{\tau}$, $(0.4 \pm 0.1) \%$ from $\tau^{-} \rightarrow K^{-} K^{+} \pi^{-} \pi^{0} \nu_{\tau}$ in $\tau^{-} \rightarrow K^{-} K^{+} \pi^{-} \nu_{\tau}$ and less than $5.0 \%$ from $\tau^{-} \rightarrow K^{-} K^{+} K^{-} \pi^{0} \nu_{\tau}$ in $\tau^{-} \rightarrow K^{-} K^{+} K^{-} \nu_{\tau}$. The non- $\tau$ backgrounds amount to less than $0.5 \%$ of the events for each channel. Detector resolution effects are removed using Bayesian Unfolding, ${ }^{8}$ which has been trained using the signal MC for each decay mode. The two-dimensional Dalitz distributions for slices of the threebody invariant mass are background subtracted and then efficiency-corrected. Simulated events are used to subtract backgrounds other than cross-feed and to correct for selection efficiency. The otbained hadronic spectra are consistent with previous measurements with smaller statistics, but deviate in some areas with respect to the high statistic Belle collaboration results. ${ }^{9}$ Fig. 4 reports the measured spectra for the $\tau^{-} \rightarrow K^{-} \pi^{+} \pi^{-} \nu_{\tau}$ channel.

\section{Acknowledgments}

We are grateful for the extraordinary contributions of our PEP-II colleagues in achieving the excellent luminosity and machine conditions that have made this work possible. The success of this project also relies critically on the expertise and 
dedication of the computing organizations that support BABAR. The collaborating institutions wish to thank SLAC for its support and the kind hospitality extended to them. This work is supported by the US Department of Energy and National Science Foundation, the Natural Sciences and Engineering Research Council (Canada), the Commissariat à l'Energie Atomique and Institut National de Physique Nucléaire et de Physique des Particules (France), the Bundesministerium für Bildung und Forschung and Deutsche Forschungsgemeinschaft (Germany), the Istituto Nazionale di Fisica Nucleare (Italy), the Foundation for Fundamental Research on Matter (The Netherlands), the Research Council of Norway, the Ministry of Education and Science of the Russian Federation, Ministerio de Ciencia e Innovación (Spain), and the Science and Technology Facilities Council (United Kingdom). Individuals have received support from the Marie-Curie IEF program (European Union) and the A. P. Sloan Foundation (USA).

\section{References}

1. J. Lees et al., Phys. Rev. D86, p. 092010 (2012).

2. A. Anastassov et al., Phys. Rev. Lett. 86, 4467 (2001).

3. J. Beringer et al., Phys. Rev. D86, p. 010001 (2012).

4. S. Nussinov and A. Soffer, Phys. Rev. D80, p. 033010 (2009).

5. J. Lees et al., Phys. Rev. D86, p. 092013 (2012).

6. P. Golonka, B. Kersevan, T. Pierzchala, E. Richter-Was, Z. Was et al., Comput. Phys. Commun. 174, 818 (2006).

7. D. Lange, Nucl. Instrum. Meth. A462, 152 (2001).

8. G. D'Agostini, Nucl. Instrum. Meth. A362, 487 (1995).

9. M. Lee et al., Phys. Rev. D81, p. 113007 (2010). 